\title{
GIST duodenal asociado a hemorragia digestiva alta. Reporte de caso
}

\author{
Héctor Losada M. ${ }^{1,2,3}$, Felipe Sanhueza V. ${ }^{1,2}$, Marcelo Klein D. ${ }^{4,5}$ y Renato Becker H. ${ }^{6,7}$
}

'Departamento de Cirugía, Traumatología y Anestesiología. Universidad de La Frontera. Temuco, Chile. ${ }^{2}$ Servicio de Cirugía Hospital Dr. Hernán Henríquez Aravena. Temuco, Chile. ${ }^{3}$ Clínica Alemana Temuco. Temuco, Chile. ${ }^{4}$ Departamento de Radiología. Hospital Dr. Hernán Henríquez Aravena. Temuco, Chile. ${ }^{5}$ Departamento de Radiología. Clínica Alemana Temuco. Temuco, Chile.

${ }^{6}$ Equipo de Anatomía Patológica. Hospital Dr. Hernán Henríquez Aravena. Temuco, Chile.

Departamento de Anatomía Patológica. Universidad de La Frontera. Temuco, Chile.

Recibido 2020-06-01 y aceptado 2020-08-06

Correspondencia a: Dr. Héctor Losada M. hector.losada@ufrontera.cl

\section{Duodenal GIST associated with upper gastrointestinal bleeding. Case report}

Introduction: Gastrointestinal stromal tumors (GIST), corresponds to $1 \%$, of all gastrointestinal neoplasms, however, only 3\%-5\% developed in duodenum. Aim: To report a case of a male patient with atypical location of gastrointestinal stroma tumor and the treatment proposed. Case report: 50-year-old male patient, with medical history of organic brain damage secondary a traumatic brain injury, smoker, occasional alcohol consumer and branquial cystomy during childhood. Consulted in the emergency department for a high digestive hemorrhage case with hemodynamic compromise. Upper digestive endoscopy, computed tomography and nuclear magnetic resonance were performed, which impresses tumor-like lesion in the second duodenal portion. Discussion: Although the GIST clinical presentation is variable, most often they are asymptomatic patients. In some times, as in this report, they may present with abdominal pain and/or upper gastrointestinal bleeding. The preoperative diagnosis was difficult, because the imaging study (CT, RNM, EDA) only stablished the suspicion and the final diagnosis was made by biopsy (we don't have endosonography in our center). Due to the anatomic repair, there is not a standardized surgery, in this case, due tumor location, infiltration and characteristics, it was decided to perform a pancreatoduodenectomy. Key words: gastrointestinal stromal tumors; gastrointestinal hemorrhage pancreatoduodenectomy.

\section{Resumen}

Introducción: Los tumores del estroma gastrointestinal (GIST) corresponden al 1\% de todas las neoplasias gastrointestinales, sin embargo, sólo el 3-5\% de estos se desarrollan en el duodeno. Objetivo: Reportar el caso de un paciente masculino con localización atípica de un tumor de estroma gastrointestinal y su manejo. Caso clínico: paciente masculino de 50 años con antecedente de traumatismo encefalo craneano (TEC) con daño orgánico cerebral secundario, tabaquismo, consumidor de alcohol ocasional y sometido a quistectomía branquial en la infancia, que consulta en el servicio de urgencias por cuadro de hemorragia digestiva alta con compromiso hemodinámico. Tras realizar endoscopia digestiva alta (EDA), resonancia nuclear magnética (RNM) y tomografía computada (TC) de abdomen, se pesquisa masa tumoral en segunda porción de duodenal. Discusión: A pesar de que la presentación clínica de los GIST es variable, lo más frecuente es que sean pacientes asintomáticos. En algunas ocasiones, al igual que en este reporte, pueden presentarse con dolor abdominal y/o hemorragia digestiva alta. El diagnóstico preoperatorio fue difícil ya que el estudio con imágenes (TC, RNM, EDA) sólo permite establecer la sospecha; el diagnóstico definitivo se realizó con biopsia (no contamos con endosonografía en nuestro centro). Debido a los sitios de reparo anatómico, no existe una cirugía estandarizada; en este caso, debido a la localización, infiltración y características, se decidió realizar una pancreatoduodenectomía.

Palabras clave: tumores del estroma gastrointestinal; hemorragia gastrointestinal; pancreatoduodenectomía.

\section{Introducción}

Los tumores del estroma gastrointestinal (GIST) corresponden al $1 \%$ de todas las neoplasias gastrointestinales, pero son la neoplasia mesenqui- matosa más frecuente del sistema digestivo. Se pueden presentar en cualquier parte del tracto gastrointestinal, con mayor frecuencia en el estómago $(60 \%)$, luego en intestino delgado $(25 \%)$, seguido de colon y recto (aproximadamente $10 \%$ ). De los 
GIST de intestino delgado, sólo un 3\%-5\% se desarrollan en el duodeno.

El diagnóstico es difícil y los estudios disponibles en la actualidad sólo permiten establecer la sospecha. Los métodos más utilizados para esto son la tomografía computarizada (TC) y la resonancia nuclear magnética (RNM). La confirmación diagnóstica se realiza histológicamente, siendo, por lo tanto, la endosonografía y biopsia el gold standard.

La resección quirúrgica es un pilar fundamental en el tratamiento del GIST duodenal, sin embargo, la desafiante ubicación anatómica con relación a sitios clave, como la cabeza del páncreas, vía biliar y vasos mesentéricos no permiten establecer un procedimiento estandarizado.

\section{Caso clínico}

Paciente de 50 años, de género masculino, con antecedente de TEC con daño orgánico cerebral secundario (hipoacusia y amaurosis de ojo derecho), hábito tabáquico con índice paquete/año (IPA) de 17 , consumo de alcohol ocasional y cirugía de quiste branquial en infancia.

Consulta en Servicio de Urgencias Adulto del
Hospital Dr. Hernán Henríquez Aravena de la ciudad de Temuco por cuadro de dolor abdominal asociado a lipotimia, hematoquexia y hematemesis no cuantificadas. Ingresa con compromiso hemodinámico. Al examen físico destacaba un abdomen doloroso a la palpación en epigastrio y al tacto rectal se constató melena.

Se realizó EDA de urgencia que informa incompetencia cardial, gastropatía congestiva córporo-antral difusa y lesión de aspecto neoplásico, ulcerada y sin sangrado activo, en la segunda porción del duodeno. Se realizó biopsia, la cual se informó sólo como duodenitis crónica inespecífica (Figura 1).

Se continuó el estudio con TC de abdomen donde destacaba gran masa exofítica en la segunda porción duodenal, de bordes bien delimitados, que compromete su pared, no pudiendo descartar si existe compromiso pancreático (Figura 2).

Se realizó RNM de abdomen en la que impresiona: Masa paraduodenal exofítica, entre la segunda y la tercera porción, de bordes bien delimitados, con captación de contraste en pared duodenal cuyas características sugieren neoplasia tipo GIST, además, impresiona compromiso de cabeza del páncreas hasta el nivel del proceso uncinado (Figura 3). A pesar de haber presentado compromiso hemodinámico,

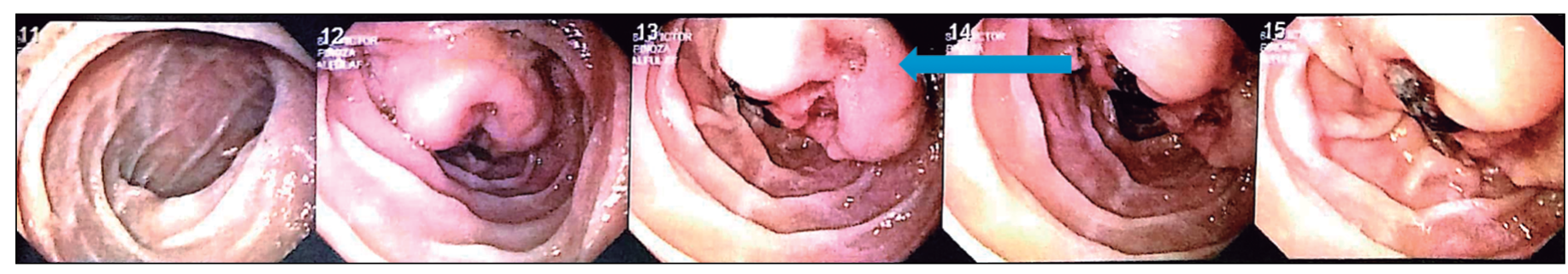

Figura 1. Endoscopía digestiva alta de urgencia, se aprecia lesión de aspecto neoplásica ulcerada en segunda porción duodenal.

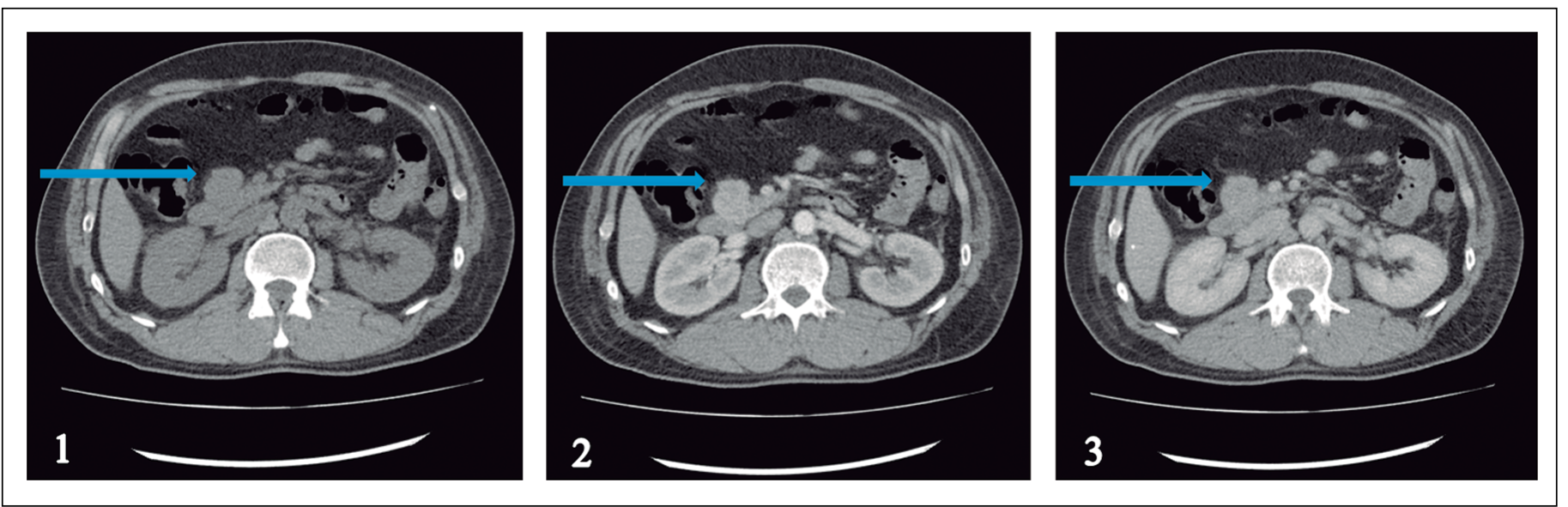

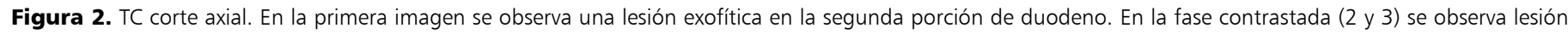

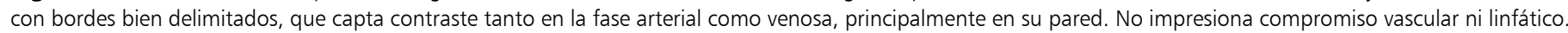


tuvo una buena respuesta al tratamiento médico de urgencias y fue dado de alta en buenas condiciones posterior al estudio. En su domicilio persistió con hemorragia de menor cuantía, requiriendo más transfusiones de glóbulo rojos (once sumando las realizadas durante hospitalización y posterior al alta). Debido a lo anterior y a las características del tumor se decide resolver de manera quirúrgica.

\section{Resultados}

Durante el acto quirúrgico se encontró un tumor intraluminal en la tercera porción del duodeno, que infiltra su pared, adherido a vena mesentérica y mesocolon transverso, que se extendía a la cabeza del páncreas, por lo cual se decidió realizar una colecistectomía y una pancreatoduodenectomía con preservación de píloro sin complicaciones y con bordes de sección negativos. La reconstrucción pancreática se realizó con pancreato-yeyunoanastomosis término-lateral. También se realizó hepatoyeyunoanastomosis término-lateral. Por último, se realiza gastro-yeyunonanastomosis.
El estudio histopatológico diferido mostró un tumor de $4 \mathrm{~cm}$ de diámetro mayor y con un índice de 3 mitosis en 50 campos de aumento mayor, estableciéndose como un GIST de bajo riesgo de malignización según la clasificación NIH modifi$\operatorname{cada}^{1-5}$ (Figura 4).

\section{Discusión}

La presentación clínica de un GIST duodenal es muy variable y depende de factores como la ubicación, la velocidad de crecimiento y el tamaño del tumor, sin embargo, la mayoría de ellos se presentan de forma asintomática siendo encontrados de forma incidental en estudios imagenológicos ${ }^{1,6,7}$.

Cuando un GIST duodenal presenta síntomas, lo más frecuente es que se manifieste como sangrado gastrointestinal en los casos en que se genera una ulceración de la mucosa, o bien, sólo como dolor abdominal. Otros síntomas, tales como saciedad precoz, ictericia u obstrucción intestinal pueden estar excepcionalmente presentes dependiendo de la ubicación del tumor ${ }^{2,6,7}$.

El diagnóstico preoperatorio es difícil y los estudios disponibles en la actualidad sólo permiten

Figura 3. RNM fase $T 1$ arterial. Corte axial. En la primera imagen se observa lesión exofítica en segunda porción duodenal, hipercaptante, que compromete la pared duodenal, con bordes bien delimitados. En la segunda imagen se observa cómo la lesión invade la cabeza del páncreas hasta el nivel del proceso uncinado.
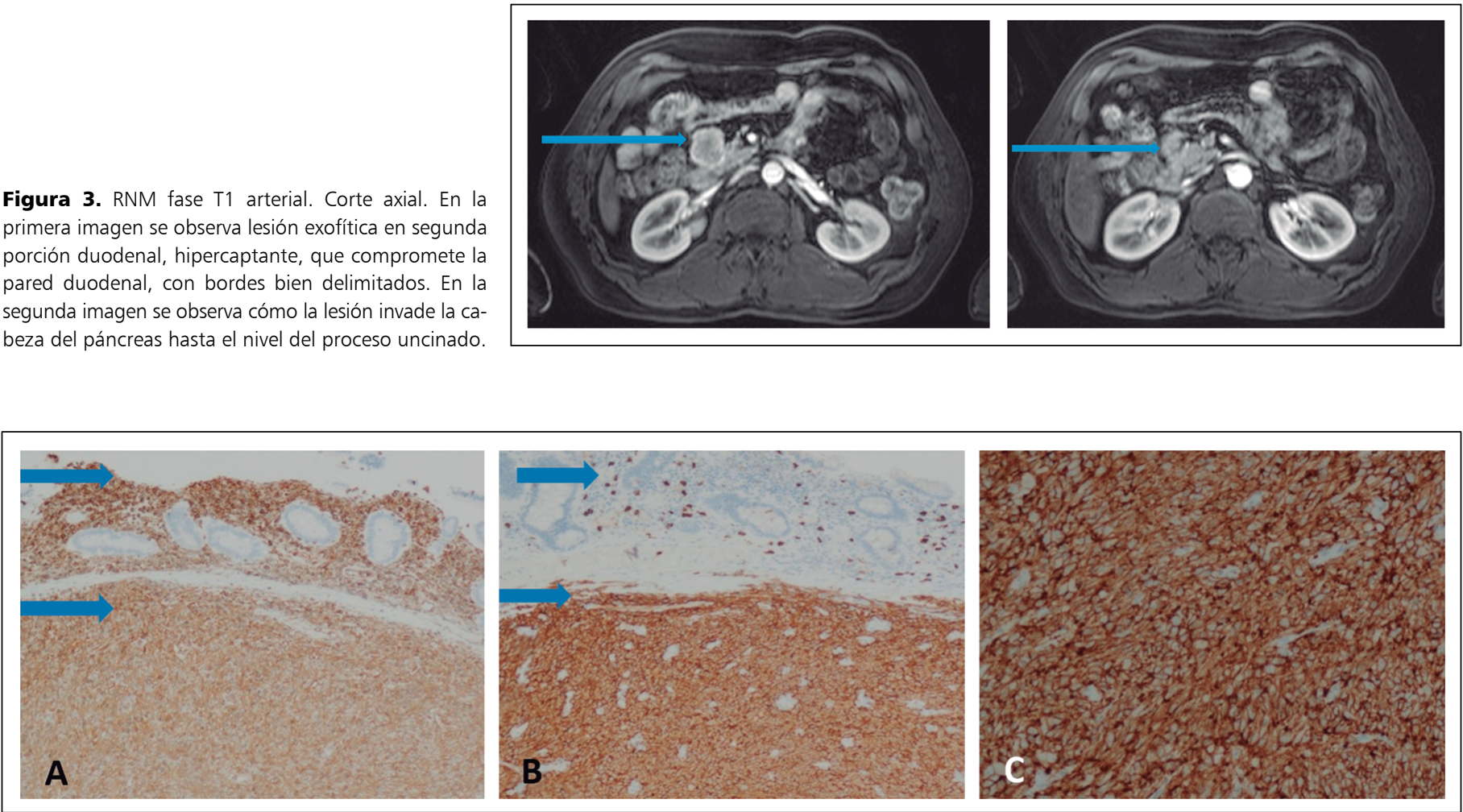

Figura 4. A: Inmunorreactividad para vimentina en células tumorales y en estroma normal de la lámina propia mucosa. B: Inmunorreactividad para CD117 en células tumorales y en escasos plasmocitos de la lámina propia. C: Inmunorreactividad para DOG1 en células tumorales. 
establecer la sospecha. Los métodos más utilizados para esto son la TC y la $\mathrm{RNM}^{1,3}$. La confirmación diagnóstica se realiza mediante histología siendo, por lo tanto, la biopsia el gold standard.

La resección quirúrgica es un pilar fundamental en el tratamiento del GIST duodenal, sin embargo, la desafiante ubicación anatómica con relación a sitios clave como la cabeza del páncreas, vía biliar y vasos mesentéricos no permiten establecer un procedimiento estandarizado. Dentro de las opciones se encuentran la resección local y la pancreatoduodenectomía. En este caso, en el acto quirúrgico, se observó una lesión exofítica ubicada entre la segunda y tercera porción del duodeno que infiltraba la cabeza del páncreas hasta el proceso uncinado por lo que se decide realizar una pancreatoduodenectomía, procedimiento más frecuentemente realizado cuando el GIST se encuentra en la segunda porción del duodeno $^{2,8-11}$. En el estudio con TC previo a la cirugía no se observaba compromiso vascular ni linfático por lo que no se realiza linfadenectomía.

En cuanto a la histología, existe la clasificación NIH modificada, la cual clasifica al GIST en 4 categorías según el riesgo de malignización: muy bajo riesgo, bajo riesgo, riesgo intermedio y alto riesgo. Esto se mide según su localización o ruptura, recuento mitótico y tamaño (Tabla 1). En este caso, la biopsia dio como resultado un tumor de origen duodenal de $4 \mathrm{~cm}$ con un índice de 3 mitosis en 50 campos de aumento mayor, por lo que clasifica como de bajo riesgo ${ }^{12}$. En el caso hipotético de que el resultado de la biopsia hubiera sido un GIST de alto riesgo, el paciente hubiese sido candidato a
Tabla 1. Clasificación NIH modificada para GIST

\begin{tabular}{|llll|}
\hline Riesgo & $\begin{array}{l}\text { Tamaño del } \\
\text { tumor }(\mathbf{c m})\end{array}$ & $\begin{array}{l}\text { Índice mitótico } \\
\text { (por campos de } \\
\text { aumento mayor) }\end{array}$ & $\begin{array}{l}\text { Localización } \\
\text { del tumor }\end{array}$ \\
Muy bajo & $>2$ & $>5 / 50$ & Cualquiera \\
Bajo & $2,1-5$ & $>5 / 50$ & Cualquiera \\
Intermedio & $>5$ & $6-10 / 50$ & Gástrico \\
& $5,1-10$ & $>5 / 50$ & Gástrico \\
Alto & Cualquiera & Cualquiera & Tumor perforado \\
& $>5$ & $>5 / 50$ & Cualquiera \\
& $>10$ & Cualquiera & Cualquiera \\
& Cualquiera & $>10 / 50$ & Cualquiera \\
& $2,1-5$ & $>5 / 50$ & No gástrico \\
& $5,1-10$ & $<5 / 50$ & No gástrico \\
\hline
\end{tabular}

terapia biológica adyuvante (imatinib, por ejemplo) posterior a la cirugía ${ }^{13}$.

\section{Responsabilidades éticas}

Protección de personas y animales. Los autores declaran que para esta investigación no se han realizado experimentos en seres humanos ni en animales.

Confidencialidad de los datos. Los autores declaran que en este artículo no aparecen datos de pacientes.

Conflictos de interés: no hay.

\section{Bibliografía}

1. Cavallaro G, Polistena A, D'Ermo G, Pedullà G, de Toma G. Duodenal gastrointestinal stromal tumors: Review on clinical and surgical aspects. International Journal of Surgery 2012;10:463-5.

2. Colombo C, Ronellenfitsch U, Yuxin Z, Rutkowski P, Miceli R, Bylina

E, et al. Clinical, Pathological and Surgical Characteristics of Duodenal Gastrointestinal Stromal Tumor and Their Influence on Survival: A Multi-Center Study. Annals of Surgical Oncology 2012;19:3361-7.

3. Mrak K, Liegl-Atzwanger B, Haybaeck
J, Petritsch W, Mischinger HJ, Kornprat P. Surgical Management of Duodenal Gastrointestinal Stromal Tumors: A Case Report. Anticancer Research 2015;35:6321-4

4. Boselli C, Cirocchi R, Gemini A, Barberini F, Grassi V, Avenia S. Urgency surgical treatment for duodenal GISTs: analysis of aged patients and review of the literature. Aging Clinical and Experimental Research 2016;29:1-6.

5. Agaimy A. Gastrointestinal stromal tumors (GIST) from risk stratification systems to the new TNM proposal: more questions than answers? A review emphasizing the need for a standardized GIST reporting. Int J Clin Exp Pathol.
2010;3:461-71

6. Melo C, Canhoto C, Manata F, Bernardes A. Surgical treatment of giant gist with acute gastrointestinal bleeding: Case report. Int J Surg Case Rep. 2018;53:3547.

7. Mulkerrin G, Hogan NM, Sheehan M, Joyce MR. Melena as an unusual presentation of gastrointestinal stromal tumour, a case report. Int J Surg Case Rep. 2018;44:172-5.

8. Krishnamurthy G, Singh H, Sharma V, Savlania A, Vasishta RK. Therapeutic Challenges in the Management of Bleeding Duodenal Gastrointestinal Stromal Tumor: a Case Report and Review of Literature. J Gastrointest 


\section{CASOS CLÍNICOS}

Cancer 2019;50:170-4.

9. Zhou Y, Wang X, Si X, Wang

S, Cai Z. Surgery for duodenal gastrointestinal stromal tumor: A systematic review and meta-analysis of pancreaticoduodenectomy versus local resection. Asian J Surg. 2020;43:1-8.

10. Popivanov G, Tabakov M, Mantese G, Cirocchi R, Piccinini I, D’Andrea V, et al. Surgical treatment of gastrointestinal stromal tumors of the duodenum: a literature review. Transl Gastroenterol Hepatol. 2018;3:71. Published online 2018 Sep 21. doi: 10.21037/tgh.2018.09.04

11. Lee SJ, Song KB, Lee YJ, Kim SC, Hwang DW, Lee JH, et al. Clinicopathologic Characteristics

and Optimal Surgical Treatment of Duodenal Gastrointestinal Stromal Tumor. J Gastrointest Surg. 2019;23:270-9.

12. Parab TM, De Rogatis MJ, Boaz AM, Grasso SA, Issack PS, Duarte DA, et al. Gastrointestinal stromal tumors: a comprehensive review. J Gastrointest Oncol. 2019;10:144-54.

13. Soriano-Lorenzo J, Lima-Pérez M, Soriano-García J, Zaldívar-Blanco K, Fleites-Calvo V. Tumores del estroma gastrointestinal. An Fac Med. [Internet]. 2019 Apr [cited 2020 July 12];80: 214-21. Disponible en: http://www.scielo.org.pe/ scielo.php?script $=$ sci_arttext\&pid=S102555832019000200015\&lng=en. 